\title{
POSITIVELY EXPANSIVE HOMEOMORPHISMS OF COMPACT SPACES
}

\author{
DAVID RICHESON and JIM WISEMAN
}

Received 15 December 2003

\begin{abstract}
We give a new and elementary proof showing that a homeomorphism $f: X \rightarrow X$ of a compact metric space is positively expansive if and only if $X$ is finite.
\end{abstract}

2000 Mathematics Subject Classification: 37B05, 37B25.

1. Introduction. A continuous map $f: X \rightarrow X$ on a metric space $X$ is positively expansive if there exists $\rho>0$ such that for any distinct $x, y \in X$ there is an $n \geq 0$ with $d\left(f^{n}(x), f^{n}(y)\right)>\rho$. The constant $\rho$ is called the expansive constant. In this note we give a simple, new proof of the following theorem.

THEOREM 1.1. Let $X$ be a compact metric space. A homeomorphism $f: X \rightarrow X$ is positively expansive if and only if $X$ is finite.

This result was first proved by Schwartzman [12]. Later, Gottschalk and Hedlund proved several results that had, as an unstated corollary, the fact that $X$ must have an isolated point (see [4, Theorems 10.30 and 10.36]). One can use this observation to prove that all points are isolated, and thus that $X$ is finite. Then Keynes and Robertson [7] gave a proof using the idea of generators for topological entropy. Later, the theorem was proved by Hiraide [6]. His proof requires a technical result of Reddy which in turn uses Frink's metrization theorem to find a compatible metric with respect to which the homeomorphism is expanding (see [2, page 41] and [3, 9]). In this note we give a proof that is short and dynamical and relies only on elementary topological arguments.

As Theorem 1.1 illustrates, positive expansiveness is a very restrictive property. One cannot restate the theorem for expansive homeomorphisms (a homeomorphism $f$ is expansive if there exists $\rho>0$ such that if $d\left(f^{n}(x), f^{n}(y)\right)<\rho$ for every integer $n$, then $x=y$ ). Although some compact spaces do not admit expansive homeomorphisms (such as the 2-sphere, the projective plane, the Klein bottle (see [5])), others do. For instance, O'Brien and Reddy proved that every compact orientable surface of positive genus admits an expansive homeomorphism (see [8]). Also, every Anosov diffeomorphism is expansive.

Furthermore, one cannot state the same theorem for noninvertible dynamical systems. For instance, the doubling map on $S^{1}$ is a positively expansive continuous map. Hiraide does prove that no positively expansive map exists on any manifold with boundary (see [6]). 
We remind the reader of some standard definitions. Let $f: X \rightarrow X$ be a homeomorphism. The $\omega$-limit set of a point $x \in X$ is defined to be

$$
\omega(x)=\bigcap_{N>0} \operatorname{cl}\left(\bigcup_{n>N} f^{n}(x)\right) .
$$

A set $S$ is invariant if $f(S)=S$. We denote the maximal invariant subset of a set $N$ by InvN. An invariant set $S$ is an isolated invariant set provided there is a compact neighborhood $N$ of $S$ with the property that $S=\operatorname{Inv} N$; the set $N$ is an isolating neighborhood for $S$. A set $S$ is an attractor if there is an isolating neighborhood $N$ for $S$ with the property that $f(N) \subset \operatorname{Int} N$ (Int $N$ is the interior of $N$ ); in this case $N$ is called an attracting neighborhood. Likewise, $S$ is a repeller if it has a repelling neighborhood, an isolating neighborhood $N$ with the property $f^{-1}(N) \subset \operatorname{Int} N$. Finally, we let $B_{\varepsilon}(x)$ denote the $\varepsilon$-ball about $x$.

2. Bounded dynamical systems. This work relies heavily on the notion of bounded dynamical systems (see [10,11]). A dynamical system is bounded if there exists a compact set $W$ with the property that the forward orbit of every point in $X$ intersects $W$. Such a set, $W$, is called a window. Clearly every dynamical system on a compact space $X$ is bounded, thus the notion of boundedness is only interesting for noncompact spaces.

Below we state several properties that are equivalent to boundedness; the theorem is proved in [10], but since the proof is short we include it again here. We note that the theorem is also true for flows or semiflows and the proof is nearly identical to the one given below.

THEOREM 2.1. If $X$ is a locally compact metric space and $f: X \rightarrow X$ is a continuous map, then the following are equivalent:

(1) $f$ is bounded;

(2) there is a compact set $V$ such that $\varnothing \neq \omega(x) \subset V$ for all $x \in X$;

(3) there exists a forward invariant window;

(4) there is a compact global attractor $\Lambda$ (i.e., there is an attractor $\Lambda$ with the property that $\varnothing \neq \omega(x) \subset \Lambda$ for every $x \in X)$.

Proof. It is clear that $(4) \Rightarrow(3) \Rightarrow(2) \Rightarrow(1)$. Thus, we must prove that the existence of a window implies the existence of a compact global attractor, (1) $\Rightarrow(4)$.

Suppose $f$ has a window $W$. It suffices to show that there is a window $W_{1}$ with the property $f\left(W_{1}\right) \subset \operatorname{Int}\left(W_{1}\right)$. For each $x \in X$, there is an $n_{x} \geq 0$ such that $f^{n_{x}}(x) \in W$. Let $\delta>0$, and let $W_{0}=\operatorname{cl}\left(B_{\delta}(W)\right)$, the closure of the $\delta$-neighborhood of $W$. Clearly, for each $x \in W_{0}, \operatorname{cl}\left(B_{\delta / 2}\left(f^{n_{x}}(x)\right)\right) \subset \operatorname{Int} W_{0}$. Moreover, there is an open neighborhood $U_{x}$ of $x$ such that $\operatorname{cl}\left(B_{\delta / 2}\left(f^{n_{x}}(y)\right)\right) \subset \operatorname{Int} W_{0}$ for all $y \in U_{x}$. The sets $\left\{U_{x}: x \in W_{0}\right\}$ form an open cover of $W_{0}$. Since $W_{0}$ is compact, there is a finite subcover, $\left\{U_{x_{1}}, \ldots, U_{x_{m}}\right\}$. Let $n=\max \left\{n_{x_{k}}: k=1, \ldots, m\right\}$. It follows that $\bigcup_{k=0}^{n} f^{k}\left(W_{0}\right)$ is a forward invariant window (thus proving (3)). However, we would like the stronger result of (4).

Consider the multivalued map $V_{r}(x)=B_{r}(x)$. By the compactness of $W_{0}$, there is an $\varepsilon>0$ such that $\left(V_{\varepsilon} \circ f\right)^{n_{x_{i}}}(y) \subset \operatorname{Int} W_{0}$ for all $y \in U_{x_{i}}$. Then, the set $W_{1}=\bigcup_{k=0}^{n}\left(V_{\varepsilon} \circ\right.$ $f)^{k}\left(W_{0}\right)$ has the desired property. 
3. Positively expansive homeomorphisms on compact spaces. In the discussion that follows it is necessary to work in the product space $X \times X$. Given a homeomorphism $f: X \rightarrow X$, we use the notation $f \times f: X \times X \rightarrow X \times X$ to denote the homeomorphism $(f \times f)\left(x_{1}, x_{2}\right)=\left(f\left(x_{1}\right), f\left(x_{2}\right)\right)$. Also, we let $\Delta=\{(x, x): x \in X\}$ denote the diagonal of $X \times X$.

It is well known that a homeomorphism $f: X \rightarrow X$ of a compact space $X$ is expansive if and only if the diagonal $\Delta$ is an isolated invariant set for $f \times f$ (see [1]). Analogously we prove that for positively expansive homeomorphisms the diagonal is a repeller.

LEMMA 3.1. Let $f: X \rightarrow X$ be a positively expansive homeomorphism of a compact space $X$. Then $\Delta$ is a repeller for $f \times f: X \times X \rightarrow X \times X$.

Proof. Suppose $X$ is a compact space and $f: X \rightarrow X$ is a positively expansive homeomorphism with expansive constant $\rho$. If $X$ is a one-point space, the conclusion of the lemma is clearly true. Thus we may assume that $X$ has at least two points. Consider the space $X \times X$ and the homeomorphism $F=f \times f$. $F$ restricts to a homeomorphism $F_{Y}: Y \rightarrow Y$, where $Y=(X \times X) \backslash \Delta$. Let $W=\left\{(x, y) \in Y: d_{X}(x, y) \geq \rho\right\}$. Clearly $W$ is a compact set and, since $f$ is positively expansive, the forward orbit of every point in $Y$ intersects $W$. Thus $W$ is a window for $F_{Y}$, and we conclude that $F_{Y}$ is bounded.

By Theorem 2.1 there exists a window $W_{1} \subset Y$ for $F_{Y}$ with $F_{Y}\left(W_{1}\right) \subset \operatorname{Int}\left(W_{1}\right)$. Then the set $N=\operatorname{cl}\left((X \times X) \backslash W_{1}\right)$ has the property that $F^{-1}(N) \subset \operatorname{Int} N$ and $\operatorname{Inv} N=\Delta$. Thus $\Delta$ is a repeller for $F$.

Proof of TheOrem 1.1. Let $f: X \rightarrow X$ be a positively expansive homeomorphism of a compact space $X$. Let $g=f^{-1}$ and $G=g \times g: X \times X \rightarrow X \times X$. By Lemma 3.1 the diagonal $\Delta \subset X \times X$ is an attractor for $G$. Thus, for $(x, y)$ sufficiently close to $\Delta$, $G^{n}(x, y) \rightarrow \Delta$ as $n \rightarrow \infty$. More precisely, there exists $\varepsilon>0$ such that if $d(x, y)<\varepsilon$, then $d\left(g^{n}(x), g^{n}(y)\right) \rightarrow 0$ as $n \rightarrow \infty$.

Define an equivalence relation $\sim$ on $X$ as follows: $x \sim y$ if and only if there exists a sequence of points $x=x_{0}, x_{1}, \ldots, x_{r}=y$ such that $d\left(x_{k}, x_{k+1}\right)<\varepsilon$ for $k=0, \ldots, r-1$. This equivalence relation is an open condition, thus each equivalence class is an open subset of $X$. Since the set of equivalence classes is a cover of $X$ by mutually disjoint open sets, the compactness of $X$ implies that there are only finitely many $U_{1}, \ldots, U_{m}$. Also, since each $U_{i}$ contains its limit points, it is closed, and hence compact.

Let $U$ be an equivalence class, and let $x, y \in U$. Then there is a sequence $x=$ $x_{0}, x_{1}, \ldots, x_{r}=y$ such that $d\left(x_{k}, x_{k+1}\right)<\varepsilon$ for $k=0, \ldots, r-1$. So, $d\left(g^{n}\left(x_{k}\right), g^{n}\left(x_{k+1}\right)\right) \rightarrow$ 0 as $n \rightarrow \infty$ for each $k=0, \ldots, r-1$. Thus $d\left(g^{n}(x), g^{n}(y)\right) \rightarrow 0$ as $n \rightarrow \infty$. Since $U$ is compact, the diameter of $g^{n}(U)$ goes to zero as $n \rightarrow \infty$.

For each $n, g^{n}\left(U_{1}\right), \ldots, g^{n}\left(U_{m}\right)$ is a collection of mutually disjoint sets whose union is all of $X$. Moreover, the diameter of each set $g^{n}\left(U_{i}\right)$ can be made arbitrarily small (letting $n$ get large). Thus, it must be the case that each $U_{i}$ consists of a single point, and that $X$ is a finite set.

ACKNOWLEDGMENT. We would like to thank Ethan Coven for bringing to our attention that Theorem 1.1 was first proved by Schwartzman [12]. 


\section{REFERENCES}

[1] E. Akin, The General Topology of Dynamical Systems, Graduate Studies in Mathematics, vol. 1, American Mathematical Society, Rhode Island, 1993.

[2] N. Aoki and K. Hiraide, Topological Theory of Dynamical Systems. Recent Advances, NorthHolland Mathematical Library, vol. 52, North-Holland Publishing, Amsterdam, 1994.

[3] A. H. Frink, Distance functions and the metrization problem, Bull. Amer. Math. Soc. 43 (1937), 133-142.

[4] W. H. Gottschalk and G. A. Hedlund, Topological Dynamics, American Mathematical Society Colloquium Publications, vol. 36, American Mathematical Society, Rhode Island, 1955.

[5] K. Hiraide, Expansive homeomorphisms of compact surfaces are pseudo-Anosov, Osaka J. Math. 27 (1990), no. 1, 117-162.

[6] _ Nonexistence of positively expansive maps on compact connected manifolds with boundary, Proc. Amer. Math. Soc. 110 (1990), no. 2, 565-568.

[7] H. B. Keynes and J. B. Robertson, Generators for topological entropy and expansiveness, Math. Systems Theory 3 (1969), 51-59.

[8] T. O'Brien and W. Reddy, Each compact orientable surface of positive genus admits an expansive homeomorphism, Pacific J. Math. 35 (1970), 737-741.

[9] W. L. Reddy, Expanding maps on compact metric spaces, General Topology Appl. 13 (1982), no. 3, 327-334.

[10] D. Richeson and J. Wiseman, A fixed point theorem for bounded dynamical systems, Illinois J. Math. 46 (2002), no. 2, 491-495.

[11]__ Bounded homeomorphisms of the open annulus, New York J. Math. 9 (2003), 55-68.

[12] S. Schwartzman, On transformation groups, Ph.D. thesis, Yale University, Connecticut, 1952.

David Richeson: Department of Mathematics and Computer Science, Dickinson College, Carlisle, PA 17013, USA

E-mail address: richesod@dickinson.edu

Jim Wiseman: Department of Mathematics and Statistics, Swarthmore College, Swarthmore, PA 19081, USA

E-mail address: jwi sema1@swarthmore.edu 


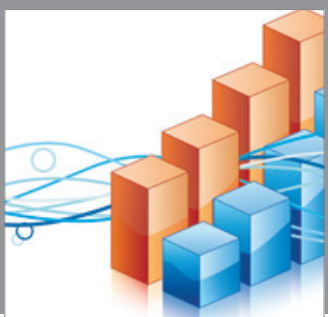

Advances in

Operations Research

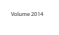

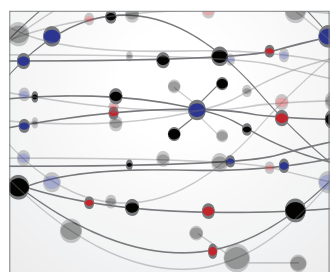

\section{The Scientific} World Journal
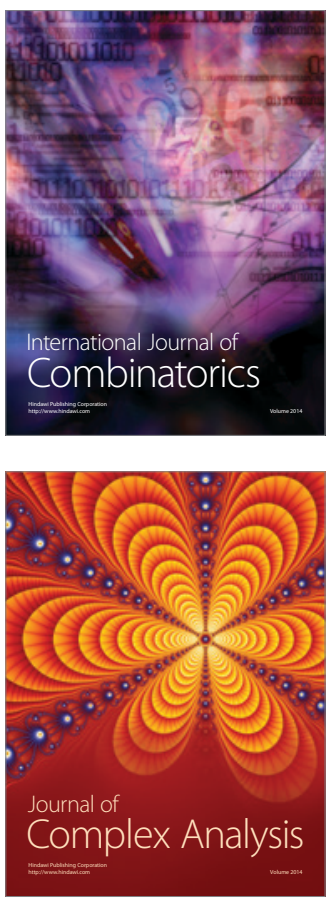

International Journal of

Mathematics and

Mathematical

Sciences
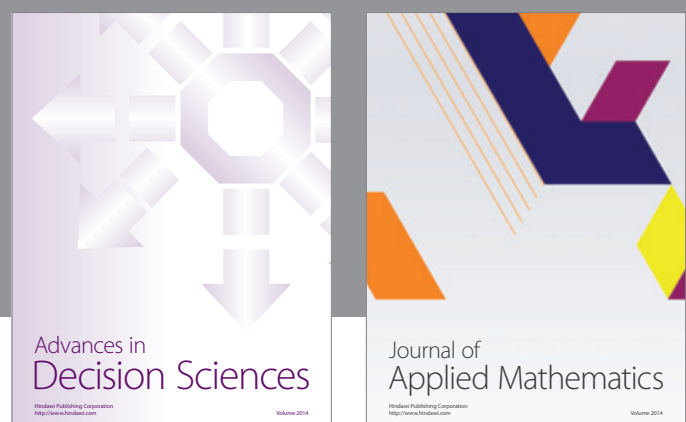

Journal of

Applied Mathematics
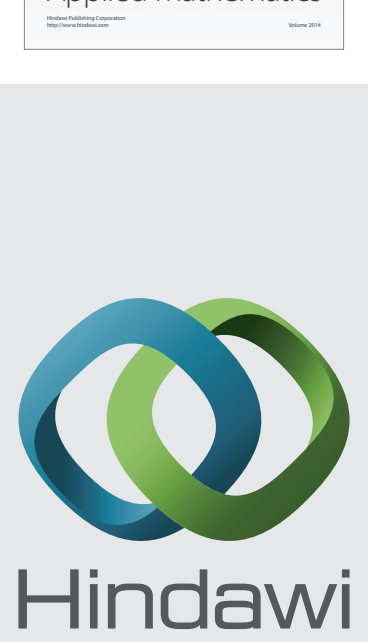

Submit your manuscripts at http://www.hindawi.com
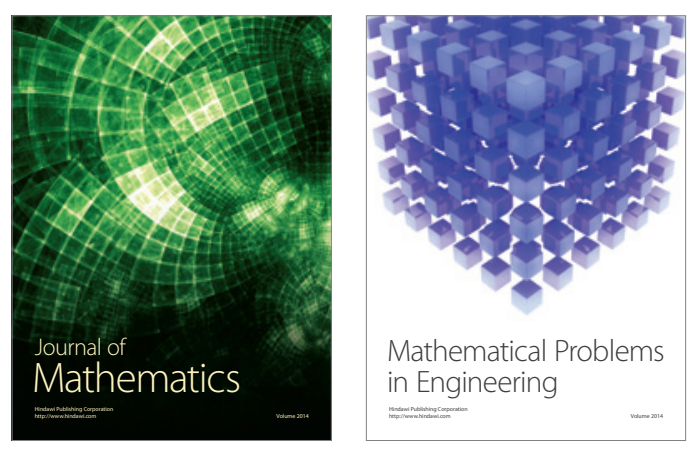

Mathematical Problems in Engineering
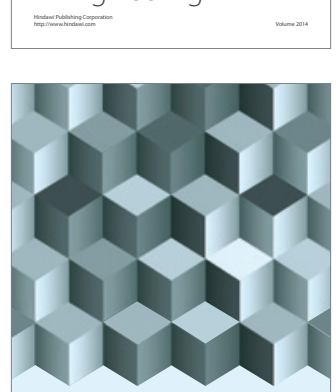

Journal of

Function Spaces
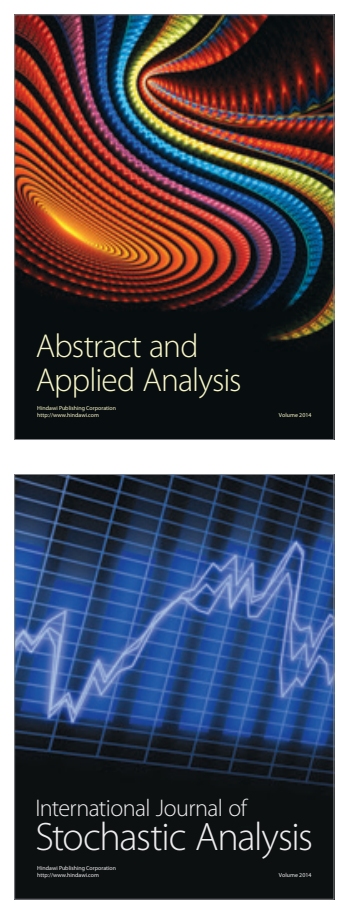

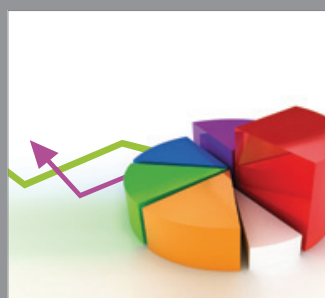

ournal of

Probability and Statistics

Promensencen
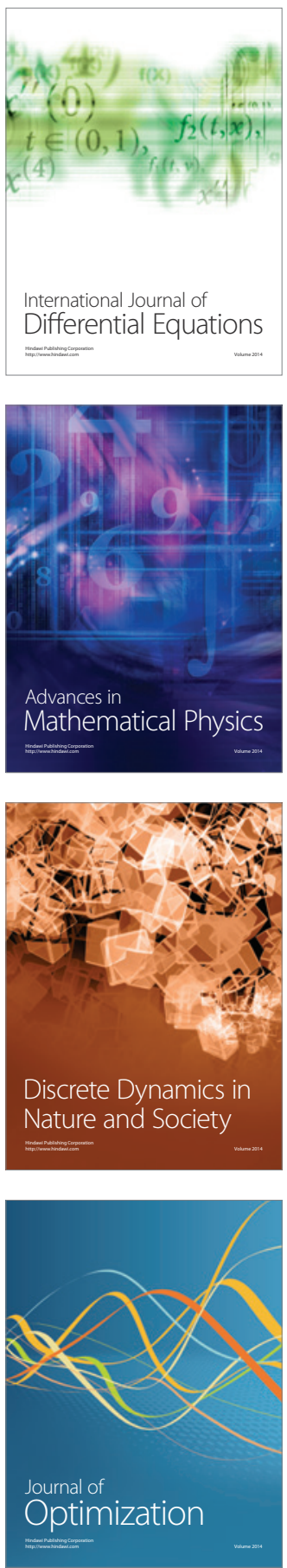